\title{
DIAGNÓSTICO DEL ESTADO ACTUALY MONITOREO DE LA CALIDAD DEL AGUA SUBTERRÁNEA DEL POZO IRHS N 179 DE LA CIUDAD UNIVERSITARIA - UNIVERSIDAD NACIONAL JORGE BASADRE GROHMANN DE TACNA
}

\author{
DIAGNOSIS OFTHE CURRENT STATUS AND MONITORING OF \\ GROUNDWATER QUALITY OFWELL IRHS N 179 UNIVERSITY CITY OF \\ NATIONAL UNIVERSITY JORGE BASADRE GROHMANN OFTACNA
}

Zoila Luz Mendoza Rodas'; Oscar Felipe Paredes Chacón $;$; María Elia Cabrera Navarrete ${ }^{3}$

\section{RESUMEN}

La Universidad Nacional Jorge Basadre Grohmann desde 1998 cuenta con un pozo de agua, con código IRHS N $N^{\circ} 179$, ubicado en la ciudad universitaria, destinada al abastecimiento para consumo humano[1], funcionamiento que se interrumpió en marzo del 2011 a consecuencia de un informe emitido por el Laboratorio de Control Ambiental DIGESA Lima[2], por el cual el agua estaría con niveles por encima de los límites permisibles de arsénico, boro, hierro y sulfatos, situación que motivo la realización del presente trabajo.

En el presente estudio se ha realizado un diagnóstico del estado actual del pozo, encontrándose inoperativo, con deficiencias técnicas en el tablero eléctrico y la bomba sumergible, con un nivel estático del agua de 77.10 metros con el último caudal registrado. Ante la imposibilidad técnica de lograr una extracción de agua del subsuelo para obtener muestras y proceder con un nuevo análisis, se procedió a realizar el monitoreo de calidad de agua a través de datos históricos registrados, desde su puesta en operación, encontrando serias incongruencias en los registros de los análisis, especialmente en los contenidos de arsénico y hierro; lo que obliga a realizar nuevos análisis en laboratorios acreditados y siguiendo los protocolos establecidos para la toma de muestras, que garanticen resultados confiables. Se realizó comparaciones en la calidad de las aguas naturales del pozo en estudio con el pozo PVI Viñani. Por la ubicación más cercana, son de naturaleza similar; sin embargo se aprecian grandes diferencias en concentración de arsénico, hierro y boro, así mismo con las aguas del río Caplina en el ingreso a la Planta de Tratamiento de Alto Lima. Las aguas en estudio superan los límites máximos permisibles por el D.S 031-2010-SA en sulfatos, dureza total, solidos disueltos, arsénico, hierro manganeso y boro.

Palabras claves: Aguas subterráneas, calidad del agua, nivel estático del agua, caudal, parámetros fisicoquímicos.

\section{ABSTRACT}

The National University Jorge Basadre Grohmann since 1998 has a water well, with code IRHS No. 179, located in the university town, for supply of water for human consumption, which was stopped in March 2011 as a result of a report issued by the Laboratory Lima DIGESA Environmental Control, for which water levels would be above the permissible limits of arsenic, boron, iron and sulfates, a situation that prompted the realization of this work.

The present study has carried out a diagnosis of the current state of the well finding inoperative with technical deficiencies with the electrical panel and submersible pump with a static water level of $77.10 \mathrm{~m}$ with the last recorded flow $121 / \mathrm{s}$. Given the technical impossibility of achieving groundwater extraction to obtain samples and conduct a new analysis, we proceeded to perform for monitoring of water quality through historical data recorded, from its start-up, finding serious inconsistencies identified in the test records, especially in the content of arsenic and iron, which requires further analysis in accredited laboratories following established protocols for sampling, to ensure reliable results. Comparisons were performed on the natural water quality of the well in studio with PVI VINANI well, for the nearest location, are of a similar nature, however great differences in concentration of arsenic, iron and boron are found, also with Caplina river waters at the entrance to the water of Alto Lima. The waters under study exceeded the maximum permissible limits for the DS 031-2010SA sulphate, total hardness, total dissolved solids, arsenic, iron, manganese and boron.

Keywords: Groundwater, water quality, static water level, flow, physicochemical parameters 


\section{I.- INTRODUCCIÓN}

El presente estudio se enmarca dentro de la principal problemática regional, cual es la escasez de recursos hídricos, debido a que Tacna se encuentra ubicada en la cabecera del desierto de Atacama. La UNJBG no es ajena a esta realidad. Para aliviar esta necesidad vital, hace cerca de 13 años que esta institución ha construido el pozo de agua subterránea, ubicado en la ciudad universitaria los Granados.

El agua extraída del subsuelo es usada directamente, sin previo tratamiento, para consumo humano en los Kioscos y cafeterías, laboratorios, servicios higiénicos y regadío de áreas verdes, de la Ciudad Universitaria, excepto para el comedor universitario que se abastece de agua potable a través de la Empresa prestadora de servicios EPS de Tacna. Ser usuarios de la red de EPS Tacna implica un costo fijo superior a los veinte mil nuevos soles[3], que nuestra institución debe desembolsar mensualmente.

\section{OBJETIVOS}

Determinar el estado actual del pozo de la CU, equipo de bombeo y tablero de control y analizar la calidad del agua de pozo, utilizada para consumo humano mediante la determinación de indicadores físicos y químicos, presencia de metales.

\section{II.-MATERIALES Y MÉTODOS}

\section{Descripción}

El presente estudio es del tipo histórico analítico.

\section{Metodología}

El año 2011 el pozo de la ciudad universitaria dejó de funcionar, debido al informe $021-$ 2001 P V I C A - E S B H A Z - D E S A DRST/GOB.REG, a raíz de los resultados de análisis fisicoquímico, indicando que el agua no era apta para el consumo humano por tener índices de arsénico y hierro muy elevados.

Posteriormente el tablero eléctrico sufrió desperfectos lo que motivó la paralización total del pozo. Debido a esta limitación, se hicieron trámites ante las autoridades de la universidad, con el objetivo de bombear el pozo y tomar muestras de agua, para su análisis, conforme a los protocolos establecidos y que garantice la efectividad y confiabilidad de los resultados. Tal intento resultó negativo. No pudiendo tomar ninguna muestra, Fue así que se procedió a trabajar con datos históricos de análisis del pozo de la C.U., para monitorear el comportamiento de las aguas a través del tiempo.

Se han considerado los datos de los resultados de análisis históricos de los años 1999 al 2010 realizados en los laboratorios del Proyecto Especial Tacna, del Ministerio de Agricultura y DIGESA.

Asumiendo la veracidad de estos datos, se procedió a hacer un estudio del comportamiento de las aguas subterráneas de este pozo a través del tiempo.

\section{III.- RESULTADOS}

El pozo IRHS $\mathrm{N}^{\circ} 179$ se encuentra ubicado en la ciudad universitaria de la Universidad Nacional Jorge Basadre Grohmann de Tacna a una altitud de $500 \mathrm{msnm}$.

Según código $\mathrm{N}^{\circ}$ IRHS $\mathrm{N}^{\circ} 179$, la fecha de inicio de perforación fue en octubre de 1998 finalizando en enero de 1999. El primer bombeo se realizó del 27 de abril al 5 de mayo de 1999. El pozo tiene una profundidad de 145 metros, se encuentra revestido con tubería ciega de 18 " hasta los $74.80 \mathrm{~m}$. con tubería ranurada de $14 \%$, desde 74 a $135 \mathrm{~m}$. y con tubería de 12", desde los 135 , hasta los $149 \mathrm{~m}$. El nivel estático del agua fue $78.45 \mathrm{~m}$. al inicio de la puesta en operación del pozo.

A la fecha de elaboración del presente trabajo, el nivel estático del agua es de $77.10 \mathrm{~m}$. La diferencia de nivel en 12 años de operaciones fue de 1 metro aproximadamente.

La última bomba sumergible la adquirió la universidad en el año 2007 es de modelo 6MQL-B09-SR6-DI-040-36-440V, marca Hidrostal, serie 2007011157 y $\varnothing$ máximo 6 pulgadas, con Motor de $40 \mathrm{HP}$, serie 07003479 , voltaje 440, A 53, RPM de $3475, \mathrm{~Hz}$ de 60 , FS:1.15, Conexión: DI, con camiseta de refrigeramiento, sin sensor de temperatura de motor; el tablero eléctrico es de marca EPLI, Potencia/ Voltaje 40/440, IP 57 amp., Arranque Soft/starter, Rampa aceleración 2 segundos, Desaceleración 2 segundos. 


\section{IV.- RESULTADOS DE LA CALIDAD DE AGUAS DEL POZO DE LA CU}

\section{Parámetros Físico Químicos del agua del Pozo de la Ciudad Universitaria}

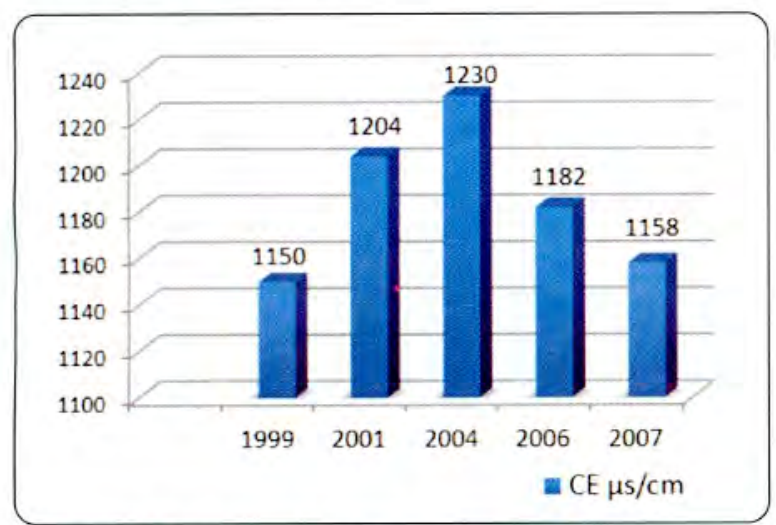

Gráfico $N^{\circ} 01$. Conductividad eléctrica.

En el Gráfico $\mathrm{N}^{\circ} 01$ se aprecia la variación de la conductividad, siendo la más alta, el año 2004, con un valor de 1230 microsiemes/cm, descendiendo a 1158 de valor para el año 2007 a 1158 .

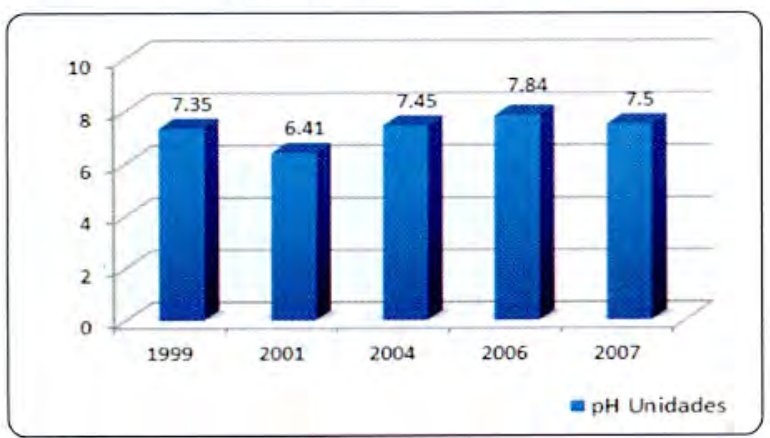

Gráfico $\mathrm{N}^{\circ}$ 02. pH

En el gráfico $\mathrm{N}^{\circ} \mathrm{O} 2$ muestra la variación del $\mathrm{pH}$ respecto al tiempo, a través de éste se observa que no ha habido variación considerable, el $\mathrm{pH}$ varia en un rango de 6,41 en el año 2001 , agua ligeramente ácida, hasta el más alto 7,84 agua ligeramente básica en el año 2006. En los análisis de los años 2008 y 2011, según DIGESA, no se reportan resultados de $\mathrm{pH}$.

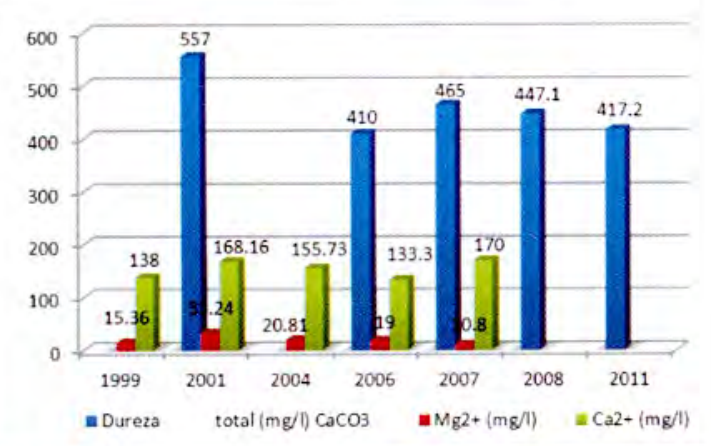

$\mathrm{El}$ agua de dureza inferior a $60 \mathrm{mgl}^{-1}$ de $\mathrm{CaCO}_{s}$ se considera blanda. Si la dureza es superior a 270 $\mathrm{mgl}^{-1}$ de $\mathrm{CaCO}_{s}$, el agua se considera dura.

En el Gráfico $\mathrm{N}^{\circ} 03$ se muestran los resultados de Dureza Total, concentración de calcio y magnesio en las 7 muestras en estudio. Se puede apreciar que los valores de dureza total fluctúan entre $410 \mathrm{mg} / \mathrm{l}$ en el año $2006 \mathrm{y}, 557 \mathrm{mg} / \mathrm{l}$, en el año 2001 ; siendo $417.2 \mathrm{mg} / \mathrm{l}$ en el 2011 ; los valores superiores a $200 \mathrm{mg} / 1$ son consideradas aguas duras, sin embargo están por debajo del máximo valor permisible $(500 \mathrm{mg} / \mathrm{l})$ la Norma del DS03 12010-SA para aguas de consumo humano; a excepción de la muestra del año 2001 que supera el rango.

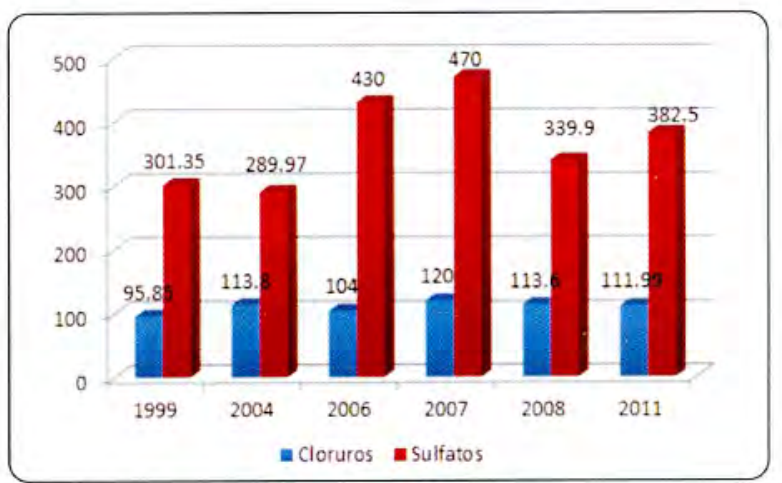

Gráfico $\mathrm{N}^{\circ}$ 04. Cloruros y sulfatos

La presencia de cloruros y sulfatos contribuyen a la salinidad de las mismas. En la Figura $\mathrm{N}^{\circ} \mathrm{O4}$ la presencia de cloruros están entre $95.85 \mathrm{mg} / \mathrm{l}$ en la muestra del año 1999 y $120 \mathrm{mg} / \mathrm{l}$ y la muestra del año 2007; siendo la muestra del 2011 de 111.99 $\mathrm{mg} / \mathrm{l}$.

Se observa que las concentraciones son similares a través del tiempo; manteniéndose dentro del límite máximo permisible de $250 \mathrm{mg} / \mathrm{l}$.

Respecto a los sulfatos, en la muestra del año 2007 aparece el máximo valor de $470 \mathrm{mg} / 1$ y el de menor valor está en la muestra del año 2004, con $289.97 \mathrm{mg} / \mathrm{l}$; observándose en la muestra del año 2011 un valor de $382.5 \mathrm{mg} / \mathrm{l}$. Todos los valores de las muestras en estudio están muy por encima del límite máximo permisible $(250 \mathrm{mg} / \mathrm{l})$ considerada en la Norma del DSo31-2010-SA para aguas de consumo humano.

En lo concerniente a sulfatos, las muestras presentan variaciones considerables a través del tiempo, lo que no se observa en cuanto al contenido de cloruros. 


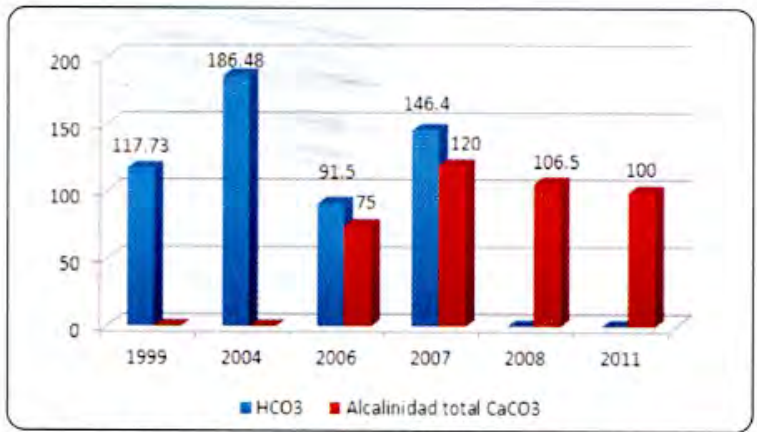

Gráfico $\mathbf{N}^{\circ}$ 05. Bicarbonatos y alcalinidad total

En el Gráfico $\mathrm{N}^{\circ} 05$ se aprecian valores entre 75 $\mathrm{mg} / \mathrm{l}$ en la muestra del año 2006 y $120 \mathrm{mg} / \mathrm{l}$ en la muestra del año 2007; las muestras de los año 1999 y 2004 no se ha determinado este parámetro; siendo la muestra del año $2011 \mathrm{de} 100 \mathrm{mg} / \mathrm{l}$.

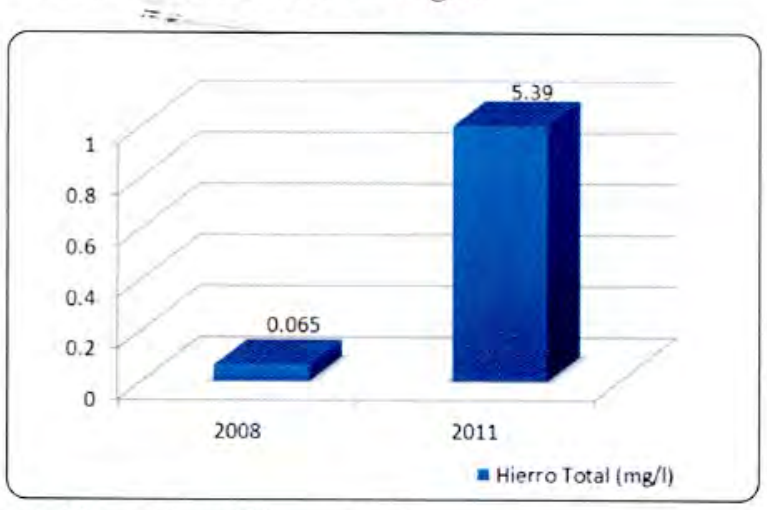

Gráfico $\mathrm{N}^{\circ}$ 06. Hierro total

En el Gráfico $\mathrm{N}^{\circ} \mathrm{O} 6$ se muestra el contenido de hierro solamente de los años 2008 y 2011 , de los anteriores años no se tiene registrado el parámetro.

En la Norma del DS031-2010-SA para aguas de consumo humano se considera como límite máximo permisible $0.3 \mathrm{mg} / 1$ para hierro. Como se puede apreciar, la muestra del año 2008 está por debajo del límite permisible, mientras que la muestra del año 2011 refleja un aumento excesivo de contenido de hierro, con un $5.39 \mathrm{mg} / 1$.

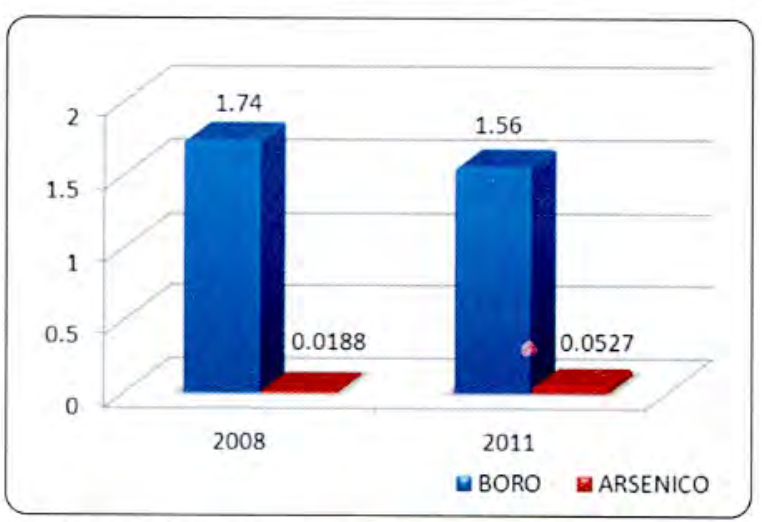

Gráfico $\mathbf{N}^{\mathbf{0}}$ 07. Contenido de Boro y Arsénico mg/l
En la Gráfico $\mathrm{N}^{\circ} 07$ se exponen las únicas dos muestras que registran resultados de análisis de Boro y Arsénico, pruebas realizadas por el laboratorio de Control Ambiental DIRESA TACNA.

El contenido de arsénico en la muestra del año 2008 fue de $0.0188 \mathrm{mg} / \mathrm{l}$, frente a la muestra del año 2011 que fue de $0.0527 \mathrm{mg} / \mathrm{l}$. Ambas muestras están fuera del límite máximo permisible y la Norma del DSo31-2010-SA para aguas de consumo humano el límiteque es de $0.01 \mathrm{mg} / \mathrm{l}$.

En la Figura $\mathrm{N}^{\circ} 07$, se muestra concentraciones de Boro; en el año 2008 fue de $1.74 \mathrm{mg} / \mathrm{l}$ y el año 2011 , de $1.56 \mathrm{mg} / \mathrm{l}$. Ambas muestras están fuera del límite máximo permisible por la Norma del DSo31-2010-SA para aguas de consumo humano que es de $1.5 \mathrm{mg} / 1$.

\section{V.DISCUSIÓN}

La comparación de la Calidad de las Aguas del Pozo de la Ciudad Universitaria con las aguas del Pozo PV 1 de Viñani y las aguas del río Caplina en el ingreso a la Planta de Tratamiento de Alto Lima es el que sigue.:

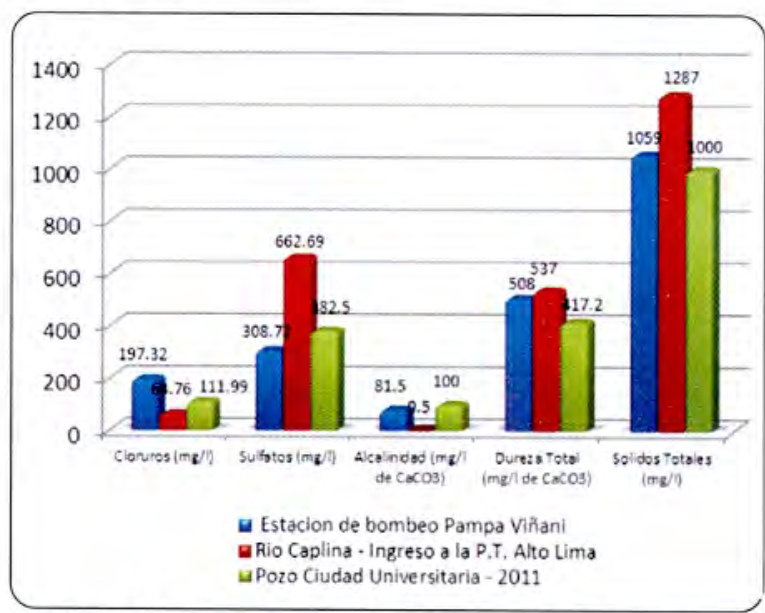

Gráfico $\mathbf{N}^{\circ}$ 08. Comparación de la Calidad de las Aguas del Pozo de la Ciudad Universitaria con las aguas del Pozo PV1 de Viñani y las aguas del río Caplina en el ingreso a la Planta de Tratamiento de Alto Lima

En el Gráfico $\mathrm{N}^{\circ} 08$ la muestra proveniente del pozo de la ciudad universitaria es la única que está dentro del límite permisible para consumo humano que es de $500 \mathrm{mg} / 1$; las otras dos muestras superan este valor; sin embargo las tres muestras son consideradas aguas duras, el contenido de cloruros es de $64.76 \mathrm{mg} / \mathrm{l}$, en la muestra que proviene del rio Caplina en su ingreso a la Planta de tratamiento de Alto lima; el pozo de la ciudad 
universitaria contiene $111.99 \mathrm{mg} / 1$ y la muestra del pozo Viñani tiene el más alto contenido de cloruro, $197.32 \mathrm{mg} / \mathrm{l}$, lo que indica que es más salina que las otras dos muestras.

En cuanto a sulfatos, la muestra que contiene mayor cantidad es el río Caplina, en el ingreso a la Planta de Tratamiento de Alto Lima, con 662.69 $\mathrm{ml} / \mathrm{g}$. El pozo de la ciudad universitaria tiene $382.5 \mathrm{mg} / 1$ y la muestra con menos sulfatos es la del pozo de Viñani PV1.

En alcalinidad el valor encontrado en el río Caplina es $0.5 \mathrm{mg} / \mathrm{l}$ y no concuerda con los valores de las otras muestras, por lo que puede considerarse un mala técnica de análisis. En el pozo de Viñani el valor de alcalinidad es de 81.5 $\mathrm{mg} / \mathrm{l}$ y en el pozo de la ciudad universitaria el valor es de $100 \mathrm{mg} / \mathrm{l}$.

El mayor contenido de sólidos totales se refleja en la muestra que proviene del río Caplina con un valor de $1287 \mathrm{mg} / \mathrm{l}$, pozo de Viñani con $1059 \mathrm{mg} / \mathrm{l}$ y el pozo de la ciudad universitaria con $1000 \mathrm{mg} / \mathrm{l}$.

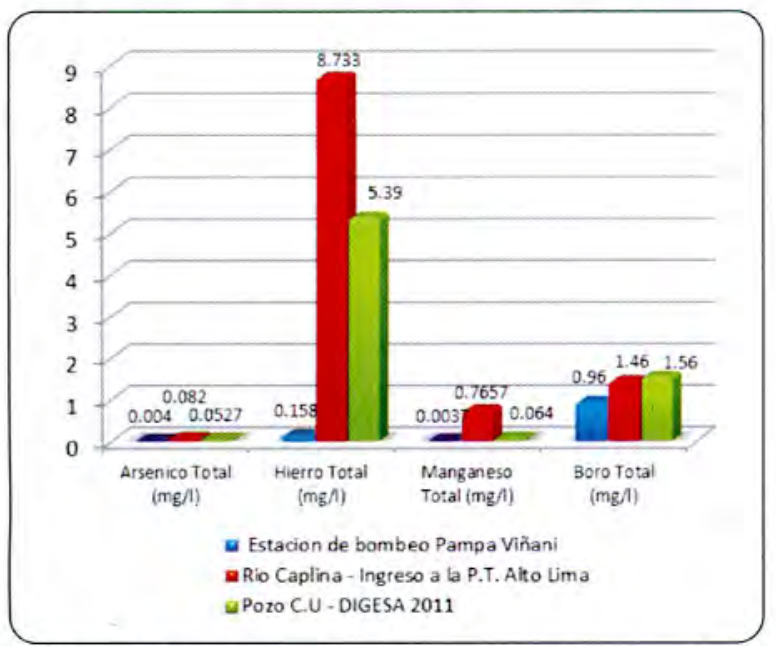

Gráfico $\mathbf{N}^{\circ}$ 09. Comparación de parámetros de calidad de agua en tres muestras (arsénico, hierro, manganeso y boro).

En el Gráfico $\mathrm{N}^{\circ} 09$ se muestra la siguiente composición del agua: el arsénico muestra una mayor concentración en la muestra del río Caplina en el ingreso a la Planta de tratamiento de Alto lima $0.082 \mathrm{mg} / \mathrm{l}$, en la muestra del pozo de la ciudad universitaria su contenido es elevado 0.0527 y la menor concentración de arsénico se presenta en la muestra del pozo de Viñani PV 1 con $0.004 \mathrm{mg} / \mathrm{l}$.

En cuanto al metal hierro, la menor concentración se presenta en el pozo de Viñani con $0.158 \mathrm{mg} / \mathrm{l}$, el pozo de la ciudad universitaria tiene $5.39 \mathrm{mg} / \mathrm{l}$ y la mayor concentración se presenta en el río Caplina, al ingreso de la Planta de Tratamiento de Alto lima, con $8.733 \mathrm{mg} / \mathrm{l}$.

El manganeso muestra su máxima concentración en el río Caplina, con $0.7657 \mathrm{mg} / \mathrm{l}$; el pozo de la ciudad universitaria tiene $0.064 \mathrm{mg} / \mathrm{l}$ y la muestra del pozo de Viñani $0.0037 \mathrm{mg} / 1$, siendo esta ultima el menor valor de manganeso.

El boro, en mayor concentración, se presenta en el pozo de la ciudad universitaria y con 1.56 $\mathrm{mg} / \mathrm{l}$; en la muestra proveniente del río Caplina, en su ingreso a la Planta de tratamiento de Alto Lima, tiene $1.46 \mathrm{mg} / 1$ de boro y el pozo de Viñani, 0.96 $\mathrm{mg} / \mathrm{l}$.

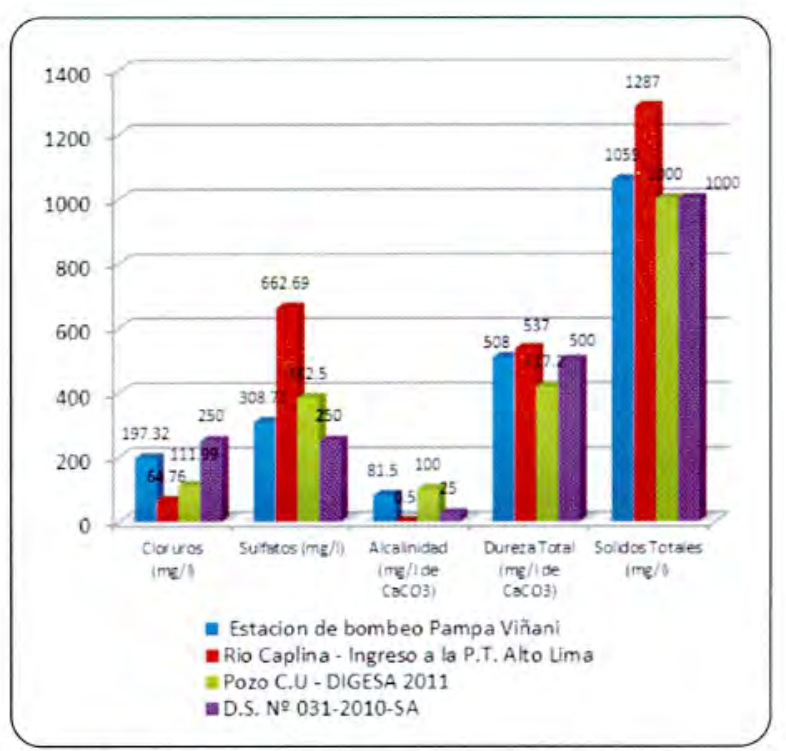

Gráfico $\mathrm{N}^{\circ} \mathbf{1 0}$. Comparación de tres muestras de aguas con las Normas del DS031-2010-SA para aguas de consumo humano (cloruros, sulfatos, alcalinidad total, dureza total y sólidos totales).

En el Gráfico $\mathrm{N}^{\circ} 10$ se comparan los valores de cloruros, sulfatos, alcalinidad total y sólidos totales, con los límites permisibles de la Norma del DS031-2010-SA, para aguas de consumo humano. Las barras de color rojo indican los valores máximos permisibles.

En cuanto a cloruros se observa claramente que las tres muestras mantienen valores menores que $250 \mathrm{mg} / \mathrm{l}$.

Referente a sulfatos, las tres muestras superan el límite permisible de $250 \mathrm{mg} / \mathrm{l}$.

En alcalinidad el límite permisible es $25 \mathrm{mg} / \mathrm{l}$. La muestra proveniente del río Caplina en el ingreso a la Planta de Tratamiento de Alto lima es de $0.5 \mathrm{mg} / \mathrm{l}$; las muestras de Viñani y el Pozo de la ciudad universitaria sobrepasan el valor máximo, con 81.5 y $100 \mathrm{mg} / \mathrm{l}$, respectivamente. 
En el contenido de dureza total, el límite permisible es de $500 \mathrm{mg} / \mathrm{l}$. Podemos observar que la única muestra que está por debajo de este valor es la del pozo de la ciudad universitaria.

En sólidos totales el pozo de la ciudad universitaria llega al límite permisible que es 1000 $\mathrm{mg} / \mathrm{l}$; las otras dos muestras superan este valor.

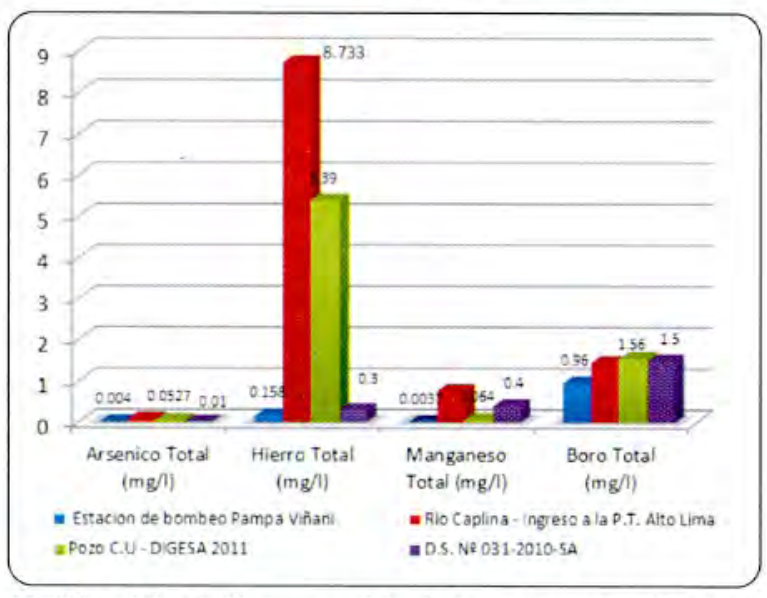

Gráfico $\mathrm{N}^{\mathbf{0}}$ 11. Comparación de tres muestras de aguas con las Normas del DS031-2010-SA para aguas de consumo humano (elementos traza).

El arsénico muestra como límite máximo permisible $0.01 \mathrm{mg} / 1$. La única muestra que está dentro de lo permitido es la del pozo de Viñani, con $0.004 \mathrm{mg} / 1$. El pozo de la ciudad universitaria y el río Caplina superan estos valores, con 0.0527 y $0.082 \mathrm{mg} / 1$ respectivamente.

En la concentración de hierro, el límite permisible es de $0.3 \mathrm{mg} / \mathrm{l}$. Las muestras del río Caplina y del pozo de la ciudad universitaria superan largamente este valor y la muestra del pozo de Viñani está dentro lo permitido, con $0.0158 \mathrm{mg} / \mathrm{l}$.

Las muestras del río Caplina superan el valor máximo para manganeso que es de $0.4 \mathrm{mg} / 1$. Las otras dos muestras en estudio están por debajo del límite aceptable.

Para el Boro, las aguas del pozo de la ciudad universitaria superan ligeramente con $1.56 \mathrm{mg} / 1$ al valor máximo permisible que es $1.5 \mathrm{mg} / 1$. Las otras dos muestras están dentro los límites para este metal.

\section{VI.- CONCLUSIONES}

- El pozo IRHS $\mathrm{N}^{\circ} 179$ de la Ciudad Universitaria de la UNJBG está inoperativo desde el mes de marzo del 2011 a la fecha,
- Presentan deficiencias en el tablero eléctrico y probablemente en la bomba sumergible.

- Debido a las serias incongruencias identificadas en los registros de los análisis de muestras de aguas del pozo de la ciudad universitaria realizados por DIGESA, especialmente por los contenidos de arsénico y hierro, será necesario hacer el análisis de una muestra del pozo de la C.U, por duplicado y en dos laboratorios diferentes, para determinar todos los parámetros considerados en la serie analítica que se muestra en el anexo, además de características organolépticas y análisis microbiológicos.

- Las aguas del pozo de la ciudad universitaria y del pozo PV1 de Viñani, por la ubicación cercana son de naturaleza similar; por lo tanto las concentraciones de los iones mayoritarios y de los elementos traza deberían ser parecidos, sin embargo, se aprecian grandes diferencias en algunos elementos como el arsénico, el hierro y boro.

\section{REFERENCIAS BIBLIOGRÁFICAS}

[1] NUÑEZ LEÓN, EDUARDO - "Estudio para la ubicación de pozos para extracción de agua subterránea en la Ciudad Universitaria”. 1998.

[2]Dirección General de Salud Ambiental Ministerio de Salud Lima-Perú Reglamento de la Calidad del Agua para Consumo Humano DS N ${ }^{\circ}$ 031-2010-SA.

[3]UNJBG - Boletín Estadístico Oficina de Planificación del 2007 al 2011.

[4] ROMERO ROJAS, JAIRO ALBERTO "Calidad del agua" - 2da Edición - Editorial Alfaomega 1999.

[5]RODIER JEAN "Análisis del agua" - 9na Edición-Editorial Omega 2011.

-6]CUSTODIO E. LLAMAS M.R - "Hidrología Subterránea" - 2da Edición - Ediciones Omega.

\section{REFERENCIAS BIBLIOGRÁFICAS}

- http://www.indecopi.gob.pe/repositorioaps /0/0/jer/acre01/Direct_Organ_Eva_Conf/ LabDeEnsayo/RelacionLab(106).pdf 
- http://www.h2ogeo.upc.es/Protocolos/

PROTOCOLS\%20i\%20GUIES/muestreo/Pr otocolo_general_IGME_UPC.pdf

-http://www.ucm.es/info/crismine/

Ambiente_Serena/Peligros_As_2.pdf

-http://dialnet.unirioja.es/servlet/articulo? codigo $=263781$

-http://ambiental.uaslp.mx/docs/FDBy

MMF-020701-NotaContVPMat.pdf

-http://www.alhsud.com/public/ebooks/

Galindo_et_al-Arsenico-2005.pdf

\section{Correspondencia:}

Zoila Luz Mendoza Rodas

Zoilaluzmr@gmail.com

Ciudad Universitaria fundo "Los Granados"

Av. Miraflores s/n Tacna - Perú

Oscar Paredes Chacón

osfepacha@yahoo.es

Ciudad Universitaria fundo "Los Granados"

Av. Miraflores s/n Tacna - Perú

María Elia Cabrera Navarrete

eliamaria@hotmail.com

Ciudad Universitaria fundo "Los Grandos"

Av. Miraflores s/n Tacna - Perú
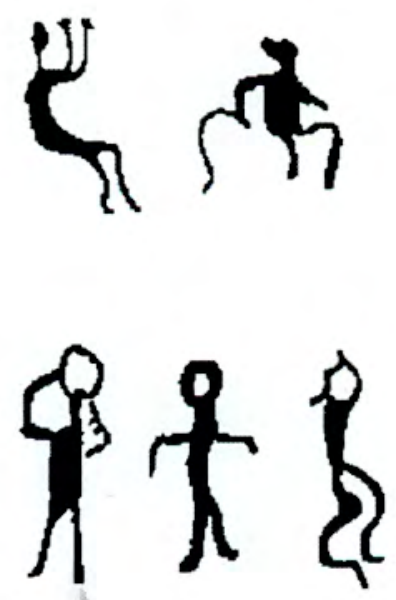